\title{
LncRNA LINC01535 promotes colorectal cancer development and chemoresistance by sponging miR-761
}

\author{
CHANGJIE ZHAO ${ }^{1}$, QI JIANG ${ }^{2}$, LIN CHEN ${ }^{2}$ and WEI CHEN ${ }^{2}$ \\ ${ }^{1}$ Endoscopy Center and ${ }^{2}$ Department of Gastroenterology, Affiliated Dongtai Hospital \\ of Nantong University, Dongtai, Jiangsu 224200, P.R. China
}

Received September 9, 2020; Accepted March 24, 2021

DOI: $10.3892 /$ etm.2021.10117

\begin{abstract}
Colorectal cancer (CRC) is one of the most common human cancer types and a leading cause of cancer-related death. Accumulating evidence has confirmed that long non-coding RNAs have crucial roles in CRC progression. In the present study, the biological roles of LINC01535 were investigated and the interaction between long intergenic non-coding RNA (LINC)01535 and microRNA (miR)-761 in CRC was explored. LINC01535 expression was observed to be upregulated in CRC tissues and cell lines. A functional study suggested that LINC01535 silencing inhibited CRC cell proliferation and invasion but enhanced cisplatin sensitivity of CRC cells, while co-transfection with a miR-761 inhibitor reversed these biological effects. A luciferase reporter assay demonstrated that LINC01535 regulated miR-761 directly and RNA-binding protein immunoprecipitation further confirmed that the suppression of LINC01535 by miR-761 was via an RNA-induced silencing complex. Finally, knockdown of LINC01535 inhibited the growth of CRC cells in vivo. Collectively, the results suggested that LINC01535 exerts oncogenic functions in CRC by sponging miR-761. In conclusion, the present study indicated that LINC01535 promoted CRC progression through sponging miR-761, and may serve as a potential diagnostic biomarker and therapeutic target for CRC.
\end{abstract}

\section{Introduction}

Colorectal cancer (CRC) has been recognized as one of the most common human cancer types and the most common causes of cancer-related death worldwide $(1,2)$. Although marked progress has been made in CRC diagnosis and treatment in the past decades, the prognosis of CRC remains rather dismal due to metastasis and recurrence (3). Therefore, further

Correspondence to: Dr Wei Chen, Department of Gastroenterology, Affiliated Dongtai Hospital of Nantong University, 2 Kangfuxi Road, Dongtai, Jiangsu 224200, P.R. China

E-mail: weichen_dthospital@21cn.com

Key words: LncRNA LINC01535 promotes colorectal cancer development and chemoresistance by sponging miR-761 exploration of the potential mechanisms underlying CRC development is urgently required to identify novel diagnostic markers and therapeutic targets for CRC.

Long noncoding RNA (lncRNA) is a class of transcripts with $>200$ nucleotides in length $(4,5)$. Accumulating evidence has indicated that IncRNAs have crucial roles in various biological processes (6-8). Furthermore, emerging studies have suggested that lncRNAs have important roles in the initiation and progression of human cancers $(9,10)$. Numerous studies have indicated that certain lncRNAs are involved in the development of CRC (11,12). Long intergenic ncRNA (LINC)01535 is a novel lncRNA that has been implicated in the development of esophageal squamous cell cancer (13), osteosarcoma (14) and cervical cancer (15). However, to date, the biological functions and molecular mechanisms of LINC01535 in CRC have remained elusive. In the present study, the expression of LINC01535 in CRC tissues and in paired adjacent normal tissues was evaluated, and the association of LINC01535 expression with clinicopathological indicators and prognosis of CRC patients was further examined. In addition, the biological roles of LINC01535 in CRC cell proliferation, invasion and cisplatin (DDP) sensitivity in vitro and tumor growth in vivo were investigated. Finally, the interaction between LINC01535 and microRNA (miRNA/miR)-761 was examined as part of elucidating the molecular mechanisms of LINC01535 in CRC in detail. To the best of our knowledge, the present study was the first to indicate the oncogenic role of LINC01535 in the development of CRC. These results offer novel insight into the progression and chemoresistance of $\mathrm{CRC}$ and may promote the development of novel anticancer therapeutic strategies for CRC.

\section{Materials and methods}

Clinical samples. A total of 24 pairs of CRC tissues and matched adjacent normal tissues were sourced from patients undergoing resection surgery at the Affiliated Dongtai Hospital of Nantong University (Dongtai, China) between January 2012 and September 2015. None of the patients received any chemotherapy or radiation therapy prior to specimen collection. All of these participants provided written informed consent prior to sample collection. The samples from resection surgery were rapidly frozen and stored in liquid nitrogen until required. This study was approved by the Ethics and Research Committees of the Affiliated Dongtai Hospital of Nantong University (Dongtai, China). 
Cell culture. Fetal human cells (FHC) from normal colonic mucosa and the human CRC cell lines HT29, LoVo, SW480 and HCT116 were purchased from the Institute of Biochemistry and Cell Biology of the Chinese Academy of Sciences. Cells were authenticated by short tandem repeat profiling. The FHC cells were maintained in Ham's F12 medium (45\%) (Gibco; Thermo Fisher Scientific, Inc.), Dulbecco's modified Eagle's medium (Gibco; Thermo Fisher Scientific, Inc.) (45\%), 25 mM HEPES, $10 \mathrm{ng} / \mathrm{ml}$ cholera toxin, $0.005 \mathrm{mg} / \mathrm{ml}$ transferrin, $0.005 \mathrm{mg} / \mathrm{ml}$ insulin, $100 \mathrm{ng} / \mathrm{Ml}$ hydrocortisone, $20 \mathrm{ng} / \mathrm{ml}$ human recombinant EGF (PHG0311; Thermo Fisher Scientific, Inc.) and fetal bovine serum (FBS, 10\%) (HyClone; Cytiva). LoVo cells were cultured in F-12K medium (Invitrogen; Thermo Fisher Scientific, Inc.). HT29, HCT116 and SW480 cells were cultured in RPMI-1640 (Gibco; Thermo Fisher Scientific, Inc.) supplemented with $10 \%$ FBS. All cells were maintained at $37^{\circ} \mathrm{C}$ with $5 \% \mathrm{CO}_{2}$ in a humidified incubator.

Constructs, synthesized oligos and transfection. Short hairpin RNA (shRNA) targeting LINC01535 (sh-LINC01535), LINC01535 (overexpression vector), miR-761 mimics, miR-761 inhibitors and their corresponding negative control (NC) were purchased from GeneChem. All of the DNAs were inserted into pcDNA3.1 (Shanghai GeneChem Co., Ltd.). Finally, Lipofectamine 3000 (Thermo Fisher Scientific, Inc.) was utilized to transfect the oligonucleotides and constructs into the SW480 and HCT116 cells according to the manufacturer's protocol. After 36-48 h of transfection, the cells were ready for the following experiments.

$R N A$ extraction and reverse transcription-quantitative $(R T-q) P C R$. Total RNA from CRC tissues and cells was extracted using TRIzol reagent (Invitrogen; Thermo Fisher Scientific, Inc.) according to the manufacturer's protocol. A total of $2 \mu \mathrm{g}$ RNA was reverse transcribed into complementary DNA using the PrimeScript RT Reagent kit (Invitrogen; Thermo Fisher Scientific, Inc.) according to the manufacturer's protocol. qPCR was performed using SYBR-Green Master Mix (Invitrogen; Thermo Fisher Scientific, Inc.) according to the manufacturer's protocol on an ABI PRISM 7500 PCR System (Applied Biosystems; Thermo Fisher Scientific, Inc.). GAPDH or U6 was used as a normalization control for mRNA and miRNA, respectively. The relative expression levels of LINC01535 and miR-761 were calculated via the $2^{-\Delta \Delta C q}$ method (16). The primer sequences are provided in Table I.

Cell proliferation assay. Cell proliferation was examined using Cell Counting Kit-8 (CCK-8) and colony formation assays. For the CCK8 assay, cells were seeded in 96-well plates at 3,000 cells/well. After 24, 48, 72 or $96 \mathrm{~h}$ of incubation, $10 \mu \mathrm{l}$ CCK-8 stain (Dojindo Molecular Technologies, Inc.) was added. After another $2 \mathrm{~h}$ of incubation, the plates were washed using PBS and the absorbance was measured at $450 \mathrm{~nm}$ with a microplate reader (ELx800; Agilent Technologies, Inc.).

Transfected DDP-resistant CRC cells (SW480/DDP and HCT116/DDP) were cultured at $37^{\circ} \mathrm{C}$ for $48 \mathrm{~h}$ prior to exposure to different doses of DDP, and then the CCK- 8 assay was performed. The $\mathrm{IC}_{50}$ was calculated using a viability curve.

For the colony formation assay, cells were seeded in a six-well plate at 1,000 cells/well and continuously cultured for 14 days, the medium was refreshed every 2-3 days. The colonies were then fixed with $4 \%$ paraformaldehyde for $10 \mathrm{~min}$ and stained with $1 \%$ crystal violet for $15 \mathrm{~min}$ at room temperature. Finally, the colonies were counted manually under a light microscope (Olympus Corp.) and images were captured.

Transwell invasion assay. Transwell invasion assays were performed to determine the cell invasion potential using Transwell plates (Corning, Inc.) that were coated with $50 \mu 1$ of Matrigel (BD Biosciences). In brief, $1 \times 10^{5}$ cells were suspended in $300 \mu \mathrm{l}$ serum-free medium and added to the upper chamber, while $800 \mu \mathrm{l}$ complete medium was placed in the lower chamber. After $24 \mathrm{~h}$ of incubation, cells on the upper surface of the membrane were scraped off. Cells on the lower side of the chamber were fixed with $100 \%$ methanol for $15 \mathrm{~min}$ at room temperature and stained with $1 \%$ crystal violet for $30 \mathrm{~min}$ at room temperature. The invading cells were counted in at least five fields under a light microscope (magnification, x200; Olympus Corp.) using ImageJ software (National Institutes of Health).

Luciferase reporter assay. The targeting interaction was predicted with a bioinformatics tool, Targetscan version 7.2 (http://www.targetscan.org/vert_72/). The wild-type (WT) and mutant-type (MUT) fragments of LINC01535 were amplified and then inserted into a pGL3 vector (Promega Corporation) to construct the luciferase reporter, referred to as LINC01535 WT and LINC01535 MUT, respectively. MUT or WT fragments of LINC01535 containing miR-761 targeting site were synthesized and cloned into a dual-luciferase reporter vector (pmirGLO; GenePharma Biotech Corp.). Luciferase vectors and miR-761 mimics or miR-761 NC together with Renilla plasmid were cotransfected into HCT116 cells by using Lipofectamine 3000. At $48 \mathrm{~h}$ after transfection, the dual-luciferase assay (Promega Corp.) was used to examine the Renilla and firefly luciferase activity following the manufacturer's protocol. The luminescence activity of firefly luciferase was normalized to that of Renilla luciferase.

RNA-binding protein immunoprecipitation (RIP) assay. The Magna RIP RNA-Binding Protein Immunoprecipitation Kit (EMD Millipore) was used for the RIP assay. Cells were harvested and lysed, and lysis buffer containing magnetic beads was incubated with human anti-Ago2 (argonaute 2) antibody (cat. no. ab186733; 1:30; Abcam) to conjugate the antibody to the magnetic beads. Subsequently, proteinase K (Sigma-Aldrich; Merck KGaA) was added to digest the protein and the immunoprecipitated RNAs were isolated using TRIzol reagent and measured by RT-qPCR as aforementioned.

Tumor xenograft experiment. HCT116 cells $\left(2 \times 10^{6}\right)$ stably transfected with sh-LINC01535 or sh-NC were subcutaneously injected subcutaneously into the left flank of 6-week-old female nude mice ( $\mathrm{n}=5$ mice per group; weight, $\sim 20 \mathrm{~g}$ ). For the animal study, humane endpoints were utilized to prevent unnecessary animal suffering according to the Guide for the Care and Use of laboratory Animals from the National Institutes of Health. In brief, the tumor burden was measured with calipers and animals were euthanized according to guidelines before the 
Table I. Primer sequences for quantitative PCR (5'-3').

\begin{tabular}{lll}
\hline Gene & \multicolumn{1}{c}{ Forward primer } & \multicolumn{1}{c}{ Reverse primer } \\
\hline LINC01535 & GGGCGGCAGGTCACTGACAC & GCCAGCAGCCGCTGGCTTAG \\
miR-761 & ACAGCAGGCACAGAC & GAGCAGGCTGGAGAA \\
GAPDH & CCAGCCGAGCCACATCGCTC & ATGAGCCCCAGCCTTCTCCAT \\
U6 & CTCGCTTCGGCAGCACATATACT & ACGCTTCACGAATTTGCGTGTC
\end{tabular}

miR, microRNA; LINC, long intergenic non-coding RNA.
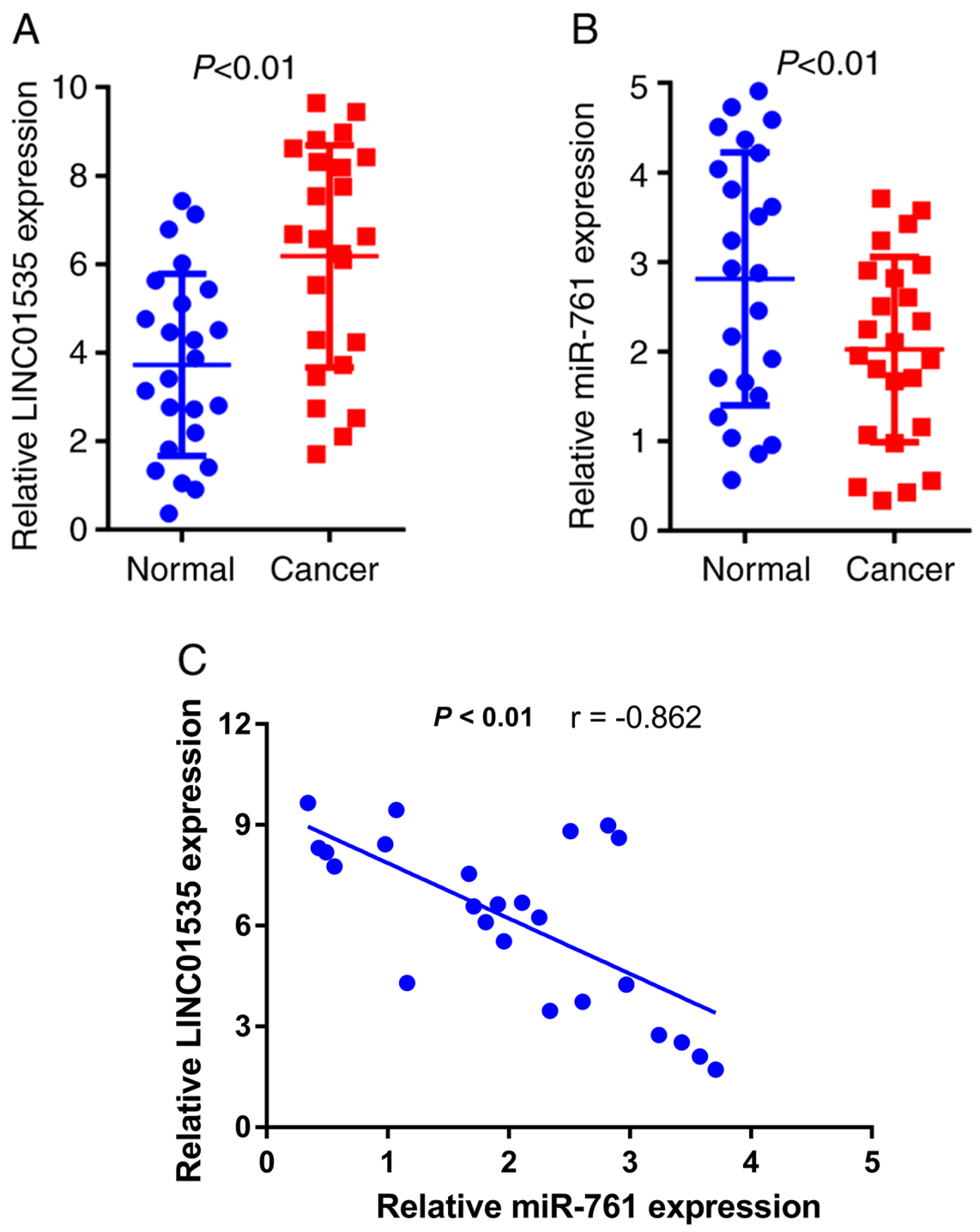

Figure 1. Expression levels of LINC01535 and miR-761 in CRC tissues. Expression of (A) LINC01535 and (B) miR-761 in 24 pairs of samples of CRC tissues and their adjacent normal tissues was evaluated by reverse transcription-quantitative PCR. (C) Correlation between LINC01535 and miR-761. miR, microRNA; LINC, long intergenic non-coding RNA; CRC, colorectal cancer.

tumor size reached the maximum permitted size (tumor diameters remained $<1 \mathrm{~cm}$ ). Regardless of the tumor size, mice were euthanized if tumors displayed ulceration, if there was distension of covering tissues, or if severe body weight loss occurred (consistent or rapid weight loss of $15 \%$ or greater compared with their initial weight). Mice were also euthanized if they displayed clinical signs necessitating immediate intervention. The mice were then placed in a carbon dioxide $\left(\mathrm{CO}_{2}\right)$ euthanasia chamber
(80 1; Shanghai Yuyan Instruments Co., Ltd.) and sacrificed by excess $\mathrm{CO}_{2}$. The controlled flow rate of $\mathrm{CO}_{2}$ was $20 \%$ of the volume of the euthanasia chamber per minute. Once the animal lost consciousness, the flow rate was increased to $100 \%$ of the euthanasia chamber volume per minute. Death was confirmed by cardiac arrest and pupil enlargement. Animal wellbeing was monitored every day and the tumor sizes were measured every week. After 4 weeks, the mice were euthanized, the 

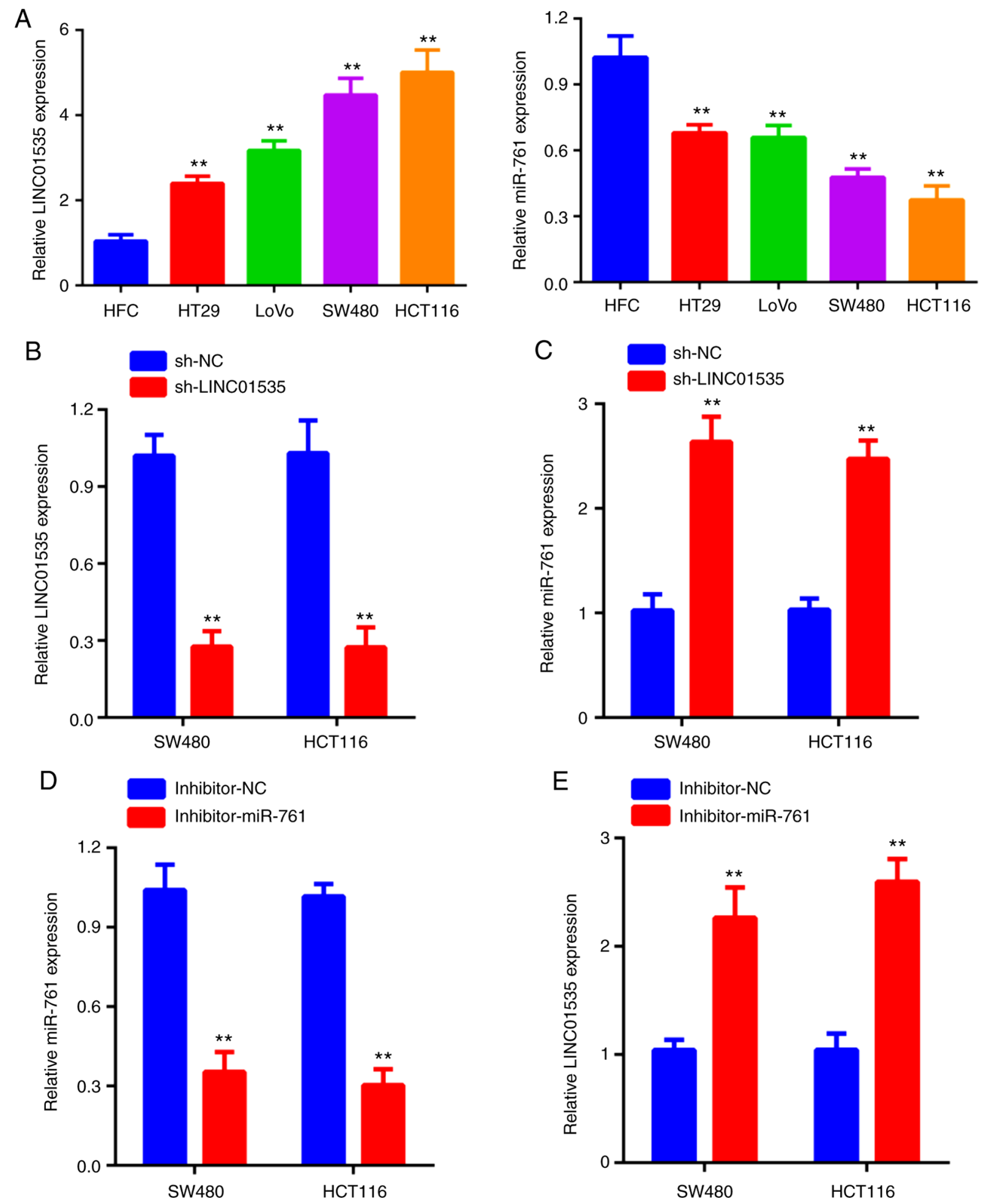

Figure 2. Negative regulatory effect of LINC01535 and miR-761. (A) The expression levels of LINC01535 and miR-761 were detected in 4 colorectal cancer cell lines and the HFC cell line. (B) SW480 and HCT116 cell lines were transfected with sh-LINC01535 or sh-NC and the knockdown efficiency was evaluated. (C) Expression levels of miR-761 in SW480 and HCT116 cells after knockdown of LINC01535. (D) RT-qPCR analysis of miR-761 after cells were transfected with miR-761 inhibitor or NC. (E) RT-qPCR analysis of LINC01535 after cells were transfected with miR-761 inhibitor or NC. ${ }^{* *} \mathrm{P}<0.01$ vs. NC/HFC. miR, microRNA; LINC, long intergenic non-coding RNA; sh-LINC01535, short hairpin RNA targeting LINC01535; NC, negative control; RT-qPCR, reverse transcription-quantitative PCR; HFC, human fetal cells.

tumor tissues were excised and weighed, and RT-qPCR was performed to determine LINC01535 and miR-761 expression. The present study was approved by the Ethics and Research Committees of the Affiliated Dongtai Hospital of Nantong University (Dongtai, China) in 2015.
Statistical analysis. Values are expressed as the mean \pm standard deviation from at least three independent experiments. One-way ANOVA followed by Tukey's post-hoc test or a two-tailed Student's t-test was performed for comparisons among multiple or between two groups, respectively. A 
A

LINC01535: gaaucuccuggguUCCUGCUGu | || | || ||

miR-761: acacagucaaaguGGGACGACg

LINC01535-mut: gaaucuccuggguGGGACGACu
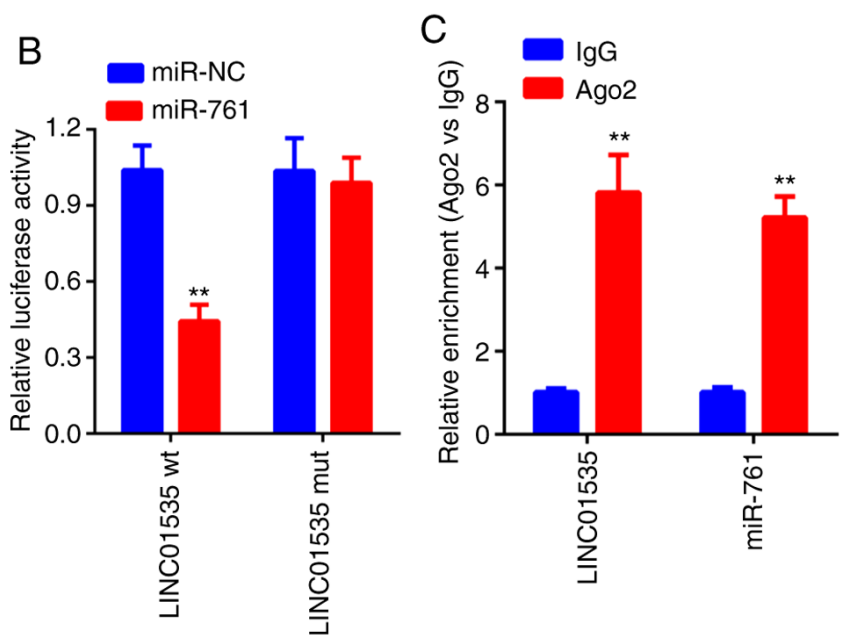

Figure 3. Direct targeting interaction of LINC01535 and miR-761. (A) Binding site of miR-761 and LINC01535 as predicted by Starbase v3.0. (B) Relative luciferase activity in HCT116 cells transfected with miR-761 mimics or mimics-NC and co-transfected with the reporter vector LINC01535-wt or LINC01535-mut. (C) RNA-binding protein immunoprecipitation assays were performed to evaluate the amount of LINC01535 bound to Ago2. ${ }^{* *} \mathrm{P}<0.01$ vs. NC/IgG. miR, microRNA; LINC, long intergenic non-coding RNA; wt, wild-type; mut, mutant; NC, negative control; Ago2, argonaute 2.

paired t-test was used for the analysis of tumor and adjacent non-tumor samples of the same individuals. The correlation of the expression of genes was assessed by calculating Pearson's correlation coefficient. $\mathrm{P}<0.05$ was considered to indicate statistical significance.

\section{Results}

LINC001535 is upregulated and miR-761 is downregulated in CRC tissues. RT-qPCR analysis was performed to determine the relative expression of LINC01535 and miR-761 in 24 pairs of CRC and adjacent non-tumor tissues. The age of the patients ranged from 33-69 years, including 11 females and 13 males (data not shown). The results indicated that LINC01535 expression was significantly upregulated (Fig. 1A), while miR-761 levels were markedly downregulated (Fig. 1B) in CRC tissues compared with those in the corresponding normal tissues. Furthermore, a significant negative correlation between LINC01535 expression and miR-761 levels in CRC tissues was determined (Fig. 1C). These results suggested that LINC01535 and miR-761 may be associated with CRC development.

Negative regulatory interaction of LINC01535 and miR-761. The result of the RT-qPCR analysis indicated that LINC01535 expression was upregulated in CRC cell lines compared with that in normal cells (human fetal cells; HFC), while the expression of miR-671 was downregulated in CRC cell lines compared with that in HFC (Fig. 2A). As presented in Fig. 2B, LINC01535 expression was markedly decreased in two CRC cell lines following transfection with sh-LINC01535. Of note, knockdown of LINC01535 significantly increased the expression of miR-761 (Fig. 2C). To further explore the correlation between LINC01535 and miR-761, SW480 and HCT116 cells were transfected with miR-761 inhibitor or corresponding control. The reduced expression of miR-761 in the two cell lines was verified by RT-qPCR (Fig. 2D). Furthermore, it was revealed that inhibition of miR-761 obviously promoted LINC01535 expression (Fig. 2E). Taken together, these results indicated the negative regulation between LINC01535 and miR-761.

miR-761 is a direct target of LINC01535. A dual-luciferase reporter assay was performed to further determine whether miR-761 is a direct target of LINC01535. The result indicated that miR-761 mimics obviously decreased the luciferase activity in the cells transfected with LINC01535-wt but not in the cells transfected with LINC01535-mut in comparison to the NC group (Fig. 3A and B). It is widely acknowledged that miRNA functions through RNA-induced silencing complex (RISC) regulation. Ago2, a key component of RISC, exerts crucial roles in RNA cleavage (17). Thus, an RIP assay was performed to determine whether miR-28 regulates LINC01535 via RISC formation. The result indicated that, compared to the NC (IgG), LINC01535 was preferentially enriched in anti-Ago2 antibody-treated beads (Fig. 3C). Collectively, these results indicated that LINC01535 directly binds to miR-761.

Effects of LINC01535 and miR-761 on CRC cell proliferation and invasion. The biological effects of LINC01535 and miR-761 on CRC cell proliferation were then explored. The results of the CCK- 8 assay suggested that knockdown of LINC01535 significantly inhibited the proliferation of SW480 and HCT16 cells but the inhibitory effect was abrogated when the cells were co-transfected with sh-LINC01535 and the miR-761 inhibitor (Fig. 4A). Similarly, the colony formation assay further confirmed that knockdown of LINC01535 significantly inhibited the proliferation of SW480 and HCT16 cells but the inhibitory effect was reversed when the cells were co-transfected with sh-LINC01535 and the miR-761 inhibitor (Fig. 4B). It was also examined whether LINC01535 and miR-761 affected CRC-cell invasion. The Transwell invasion assay suggested that silencing of LINC01535 suppressed the invasive ability of CRC cells. Similar to the above, this effect was also abrogated when sh-LINC01535 and the miR-761 inhibitor were co-transfected (Fig. 4C).

LINC01535 knockdown enhances DDP sensitivity of CRCcells. To further investigate the effect of LINC01535 and miR-761 on the DDP sensitivity of CRC cells, two DDP-resistant CRC cell lines were established (SW480/DDP and HCT116/DDP). For SW480/DDP and HCT116/DDP cells, higher IC $_{50}$ values than those in the corresponding parental cell lines SW480 and HCT116 were obtained, indicating an increased resistance to DDP (Fig. 5A). Furthermore, RT-qPCR was used to measure LINC01535 expression in parental and DDP-resistant CRC cells. Significantly increased LINC01535 expression was observed in SW480/DDP and HCT116/DDP cells (Fig. 5B). Furthermore, LINC01535 knockdown in SW480 and HCT116 cells resulted in significantly lower $\mathrm{IC}_{50}$ values than those for the control group, and this effect was reversed by miR-761 inhibitor (Fig. 5C). Taken together, these results suggested that LINC01535 induces DDP resistance in CRC cells. 


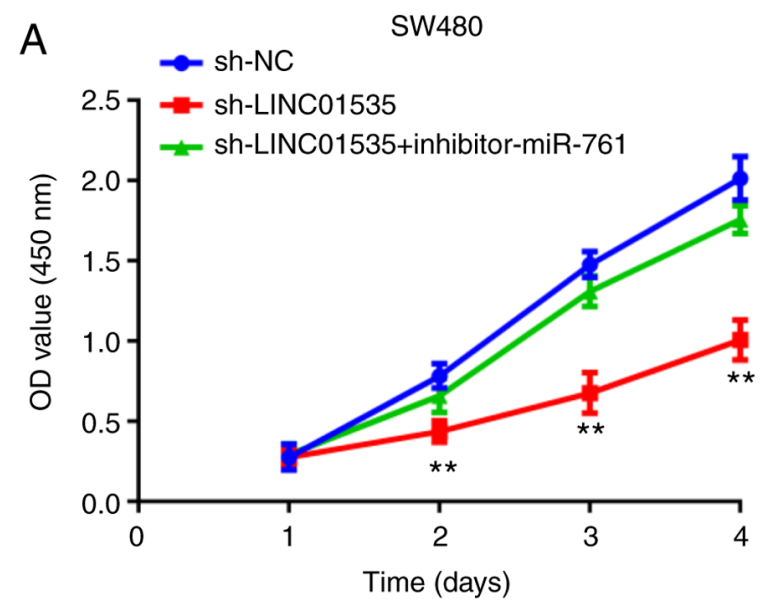

B

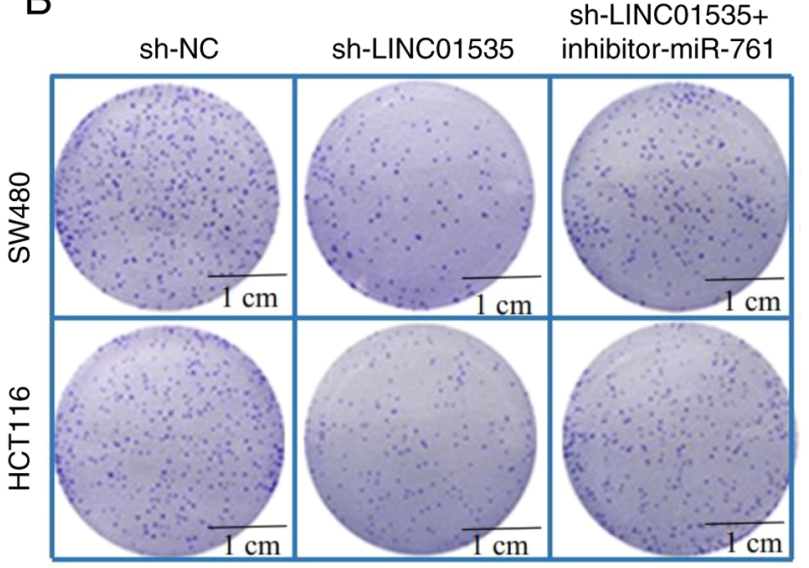

C

sh-NC
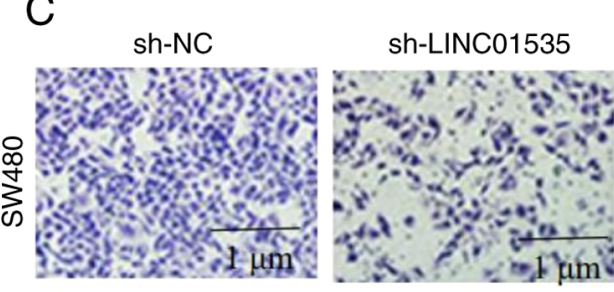

sh-LINC01535+ inhibitor-miR-761
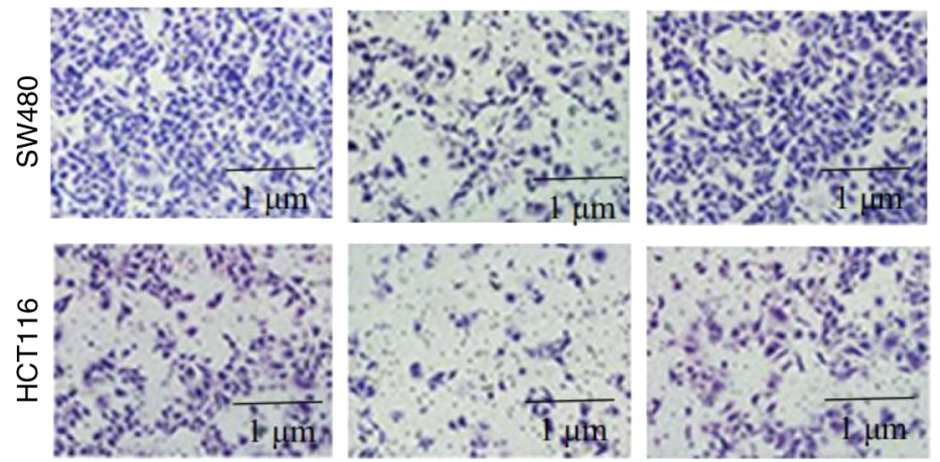
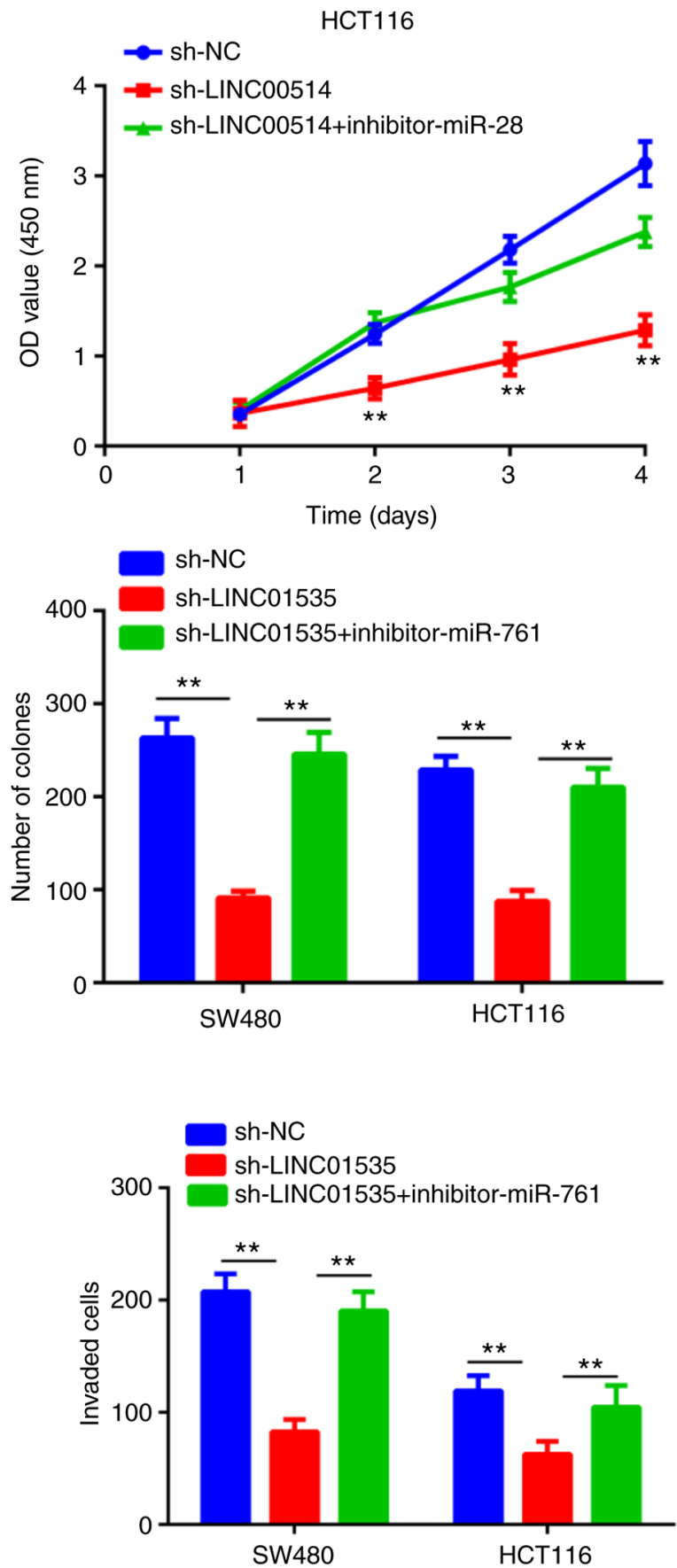

Figure 4. Roles of LINC01535 and miR-761 in colorectal cancer cell proliferation and invasion. (A) A Cell Counting Kit-8 cell viability assay was used to evaluate the proliferation in the sh-NC, sh-LINC01535 and sh-LINC01535+miR-761 inhibitor groups from days 1-4 after inoculation. (B) Colony formation assays were used to evaluate cell migration in the sh-NC, sh-LINC01535 and sh-LINC01535+miR-761 inhibitor groups (scale bars, $1 \mathrm{~cm}$ ). (C) Transwell assays were used to evaluate the invasion ability of sh-NC, sh-LINC01535 and sh-LINC01535+miR-761 inhibitor groups (scale bars, $1 \mu \mathrm{m}$ ). ${ }^{* *} \mathrm{P}<0.01 \mathrm{vs}$. NC. miR, microRNA; LINC, long intergenic non-coding RNA; sh-LINC01535, short hairpin RNA targeting LINC01535; NC, negative control; OD, optical density.

Overexpression of LINC01535 promotes CRC cell proliferation, invasion and decreases DDP sensitivity of CRC cells. As demonstrated in Fig. 6A, following transfection with LINC01535 overexpression vector, LINC01535 levels markedly increased in both cell lines compared with those in the negative control group. Furthermore, overexpression of LINC01535 enhanced the colony forming ability of SW480 and HCT116 cells (Figs. 6B and S1), as well as their invasive capacity (Figs. 6C and S1). In addition, overexpression of LINC01535 in SW480 and HCT116 cells resulted in significantly higher
$\mathrm{IC}_{50}$ values for DDP than those for the control group, further suggesting that LINC01535 induces DDP resistance in CRC cells.

LINC01535 silencing inhibits CRC tumor growth in vivo. To further verify the in vitro results, a subcutaneous xenograft tumor model was established by injecting HCT116 cells stably transfected with sh-NC or sh-LINC01535 into nude mice. Consistent with the in vitro findings, the in vivo study demonstrated that the volume and weight of tumors in the 

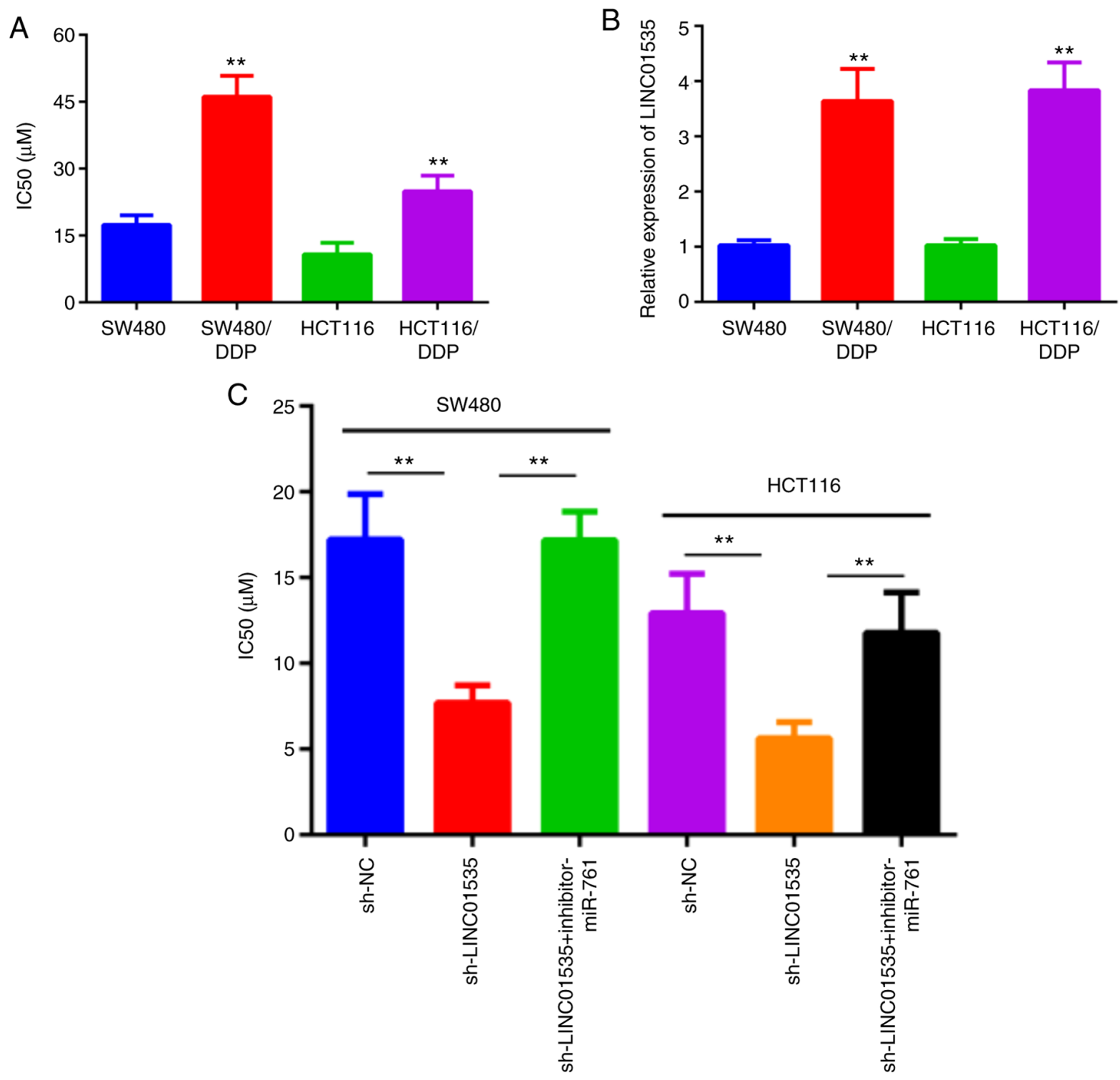

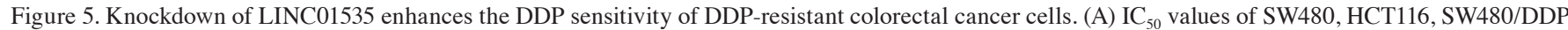
and HCT116/DDP cells for DDP for $48 \mathrm{~h}$. (B) Differential expression of LINC01535 in SW480 and HCT116 cells and the DDP-resistant SW480/DDP and HCT116/DDP cells. (C) DDP sensitivity of SW480 and HCT116 cells that were stably transfected with sh-LINC01535 and miR-761 mimics or NC. ** P<0.01. LINC, long intergenic non-coding RNA; DDP, cisplatin; SW480/DDP, SW480 cell line with acquired DDP resistance; IC $_{50}$, concentration leading to $50 \%$ cell inhibition; sh-LINC01535, short hairpin RNA targeting LINC01535; NC, negative control; miR, microRNA.

sh-LINC01535 group were significantly reduced compared with those in the sh-NC group (Fig. 7A-C). Furthermore, in the tumors of the sh-LINC01535 group, the expression of LINC01535 was decreased, while miR-761 expression was increased compared with that in the sh-NC group (Fig. 7D and E). Therefore, it was concluded that LINC01535 knockdown inhibited CRC tumor growth in vivo.

\section{Discussion}

Tumor cell proliferation, invasion and chemoresistance are important types of aggressive behavior of human cancers $(18,19)$. A large number of studies have revealed that lncRNAs may function as promoters or inhibitors of the proliferation, invasion and chemoresistance of human cancer cells $(20,21)$. It has been widely acknowledged that numerous lncRNAs are involved in the progression of
CRC. For instance, LINC00963 promotes cell proliferation and migration via the miR-124-3p/frizzled class receptor 4 signaling pathway in CRC (22). LncRNA UCID was reported to promote CRC cell migration and invasion by sponging miR-152-3P and activation of the Wnt/ $\beta$-catenin signaling pathway (23). LncRNA heart and neural crest derivatives expressed 2-antisense 1 reduced 5-fluorouracil resistance of CRC cells through regulating the miR-20a/programmed cell death 4 axis (24). However, the exact functions and underlying mechanisms of the majority of lncRNAs in CRC have remained to be elucidated.

LINC01535, a newly identified IncRNA, was reported to promote cervical cancer cell proliferation, migration and invasion in vitro and tumor growth in vivo via modulating the miR-214/enhancer of zeste 2 polycomb repressive complex 2 subunit feedback loop (15). LINC01535 was also reported to facilitate cell proliferation and inhibit apoptosis in esophageal 
A
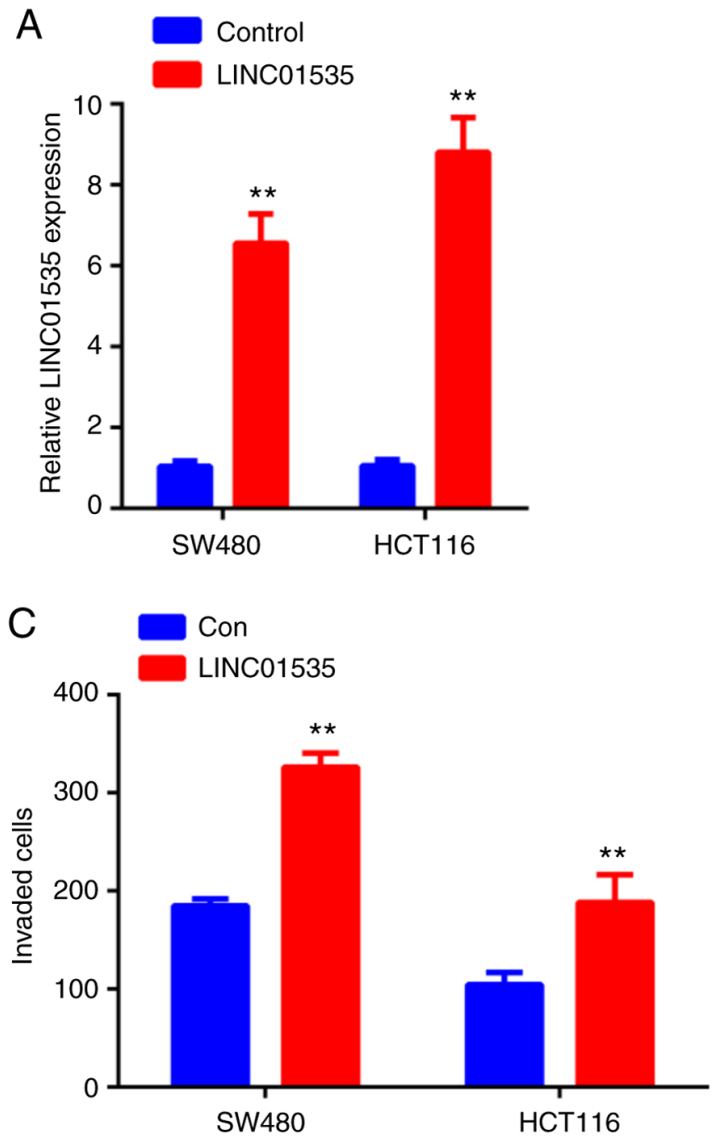

$\mathrm{B}$
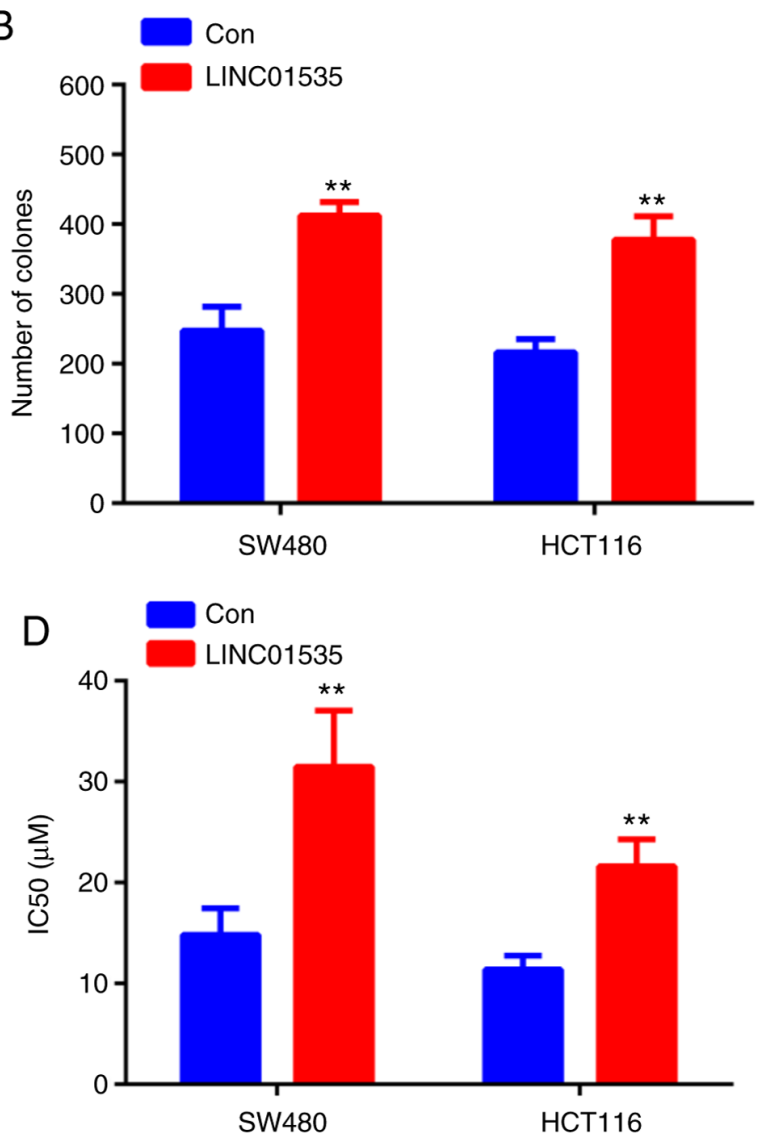

Figure 6. Overexpression of LINC01535 promotes CRC cell proliferation and invasion and inhibits DDP sensitivity. (A) SW480 and HCT116 cell lines were transfected with LINC01535 or control plasmid. (B) Colony formation assays were used to evaluate the effects of LINC01535 overexpression on CRC proliferation. (C) Transwell invasion assays were used to evaluate the effects of LINC01535 overexpression on CRC invasion. (D) Overexpression of LINC01535 reduced the DDP sensitivity of CRC cells. ${ }^{* *} \mathrm{P}<0.01$ vs. Con. LINC, long intergenic non-coding RNA; DDP, cisplatin; CRC, colorectal cancer; Con, control; $\mathrm{IC}_{50}$, concentration leading to $50 \%$ cell inhibition.

squamous cell carcinoma through activating the JAK/STAT3 signaling pathway (13). In addition, LINC01535 was indicated to promote osteosarcoma progression via regulating the miR-214-3p/potassium voltage-gated channel subfamily C member 4 axis (14). In the present study, it was determined that the expression of LINC01535 was significantly increased in CRC tissues and cell lines. Considering the relatively small number of included patients, the association between LINC001535 expression and the clinicopathological characteristics of patients with CRC was not explored. Furthermore, an in vitro functional study demonstrated that silencing of LINC01535 suppressed cell proliferation and invasion. In addition, the in vivo results confirmed that LINC01535 knockdown inhibited CRC growth in a xenograft model. DDP is a classic and effective cell cycle non-specific anti-cancer drug, which has been widely used to treat a variety of solid malignancies, including CRC $(25,26)$. Patients with CRC frequently display good initial responses to DDP-based chemotherapy; however, DDP resistance usually occurs. Therefore, enhancing DDP sensitivity in DDP-resistant CRC cells is extremely important for the treatment of CRC (27). The present study provides the first evidence, to the best of our knowledge, that LINC01535 knockdown enhanced DDP sensitivity in DDP-resistant CRC cells.

Accumulating studies suggested that IncRNAs exert their functions through binding with miRNAs as competing endogenous (ce)RNAs (28). For instance, LINC02418 upregulated maternal embryonic leucine zipper kinase expression by acting as a ceRNA in CRC (29). LncRNA HOX transcript antisense RNA knockdown enhanced radiosensitivity through regulating the miR-93/autophagy related 12 axis in CRC (30). In the present study, an online database was used to identify potential target miRNAs of LINC01535 and miR-761 was selected. Previous studies have established the inhibitory function of miR-761 in various human cancer types, including colorectal cancer (31), gastric cancer (32) and ovarian cancer (33). In LINC01535-knockdown CRC cells, the expression of miR-761 was significantly increased. In addition, the expression of miR-761 was negatively associated with LINC01535 expression in CRC tissues. The results of the luciferase reporter and RIP assay further confirmed that LINC01535 directly bound to miR-761. Finally, the results of the functional rescue assay indicated that LINC01535 knockdown inhibited CRC cell proliferation and invasion and enhanced DDP sensitivity through regulating miR-761. Additionally, there are some limitations that should be addressed. Firstly, in the animal experiments, DDP could have been used in some additional groups to determine the effect of LINC01535 on DDP resistance in vivo. Furthermore, in a future study, the sample size should be increased to evaluate the association of LINC01535 and 


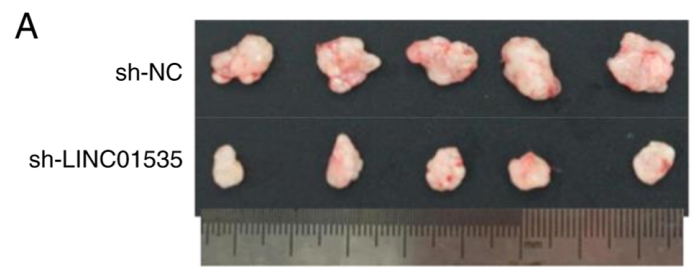

B

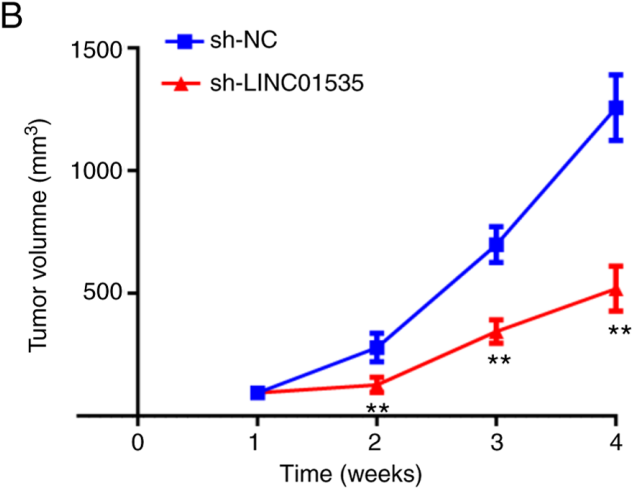

D

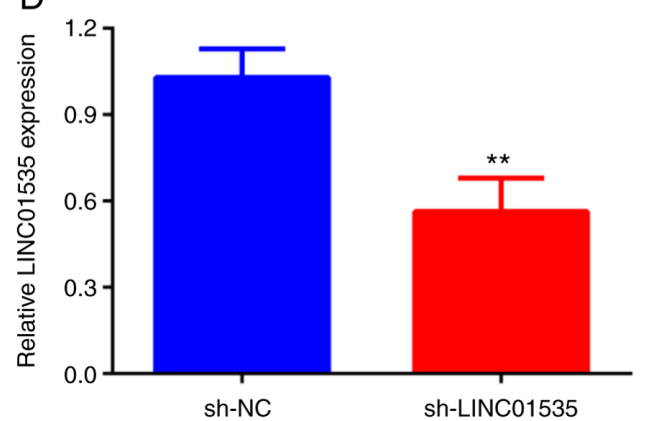

C

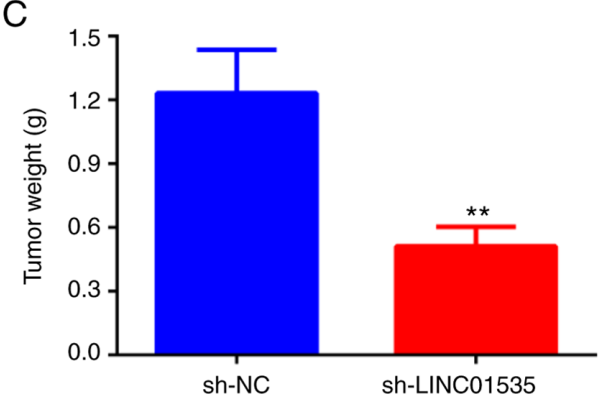

$\mathrm{E}$

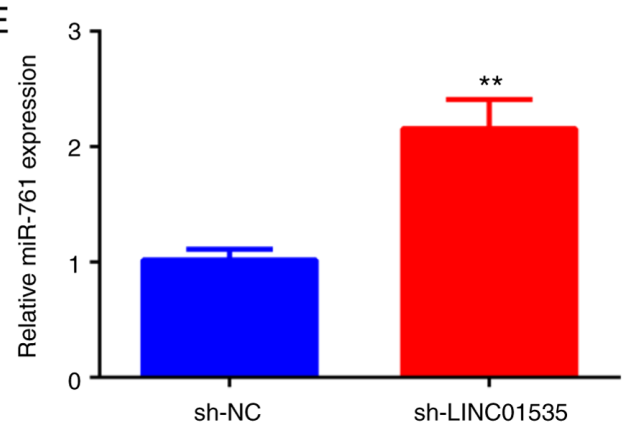

Figure 7. Knockdown of LINC01535 inhibits colorectal cancer growth in vivo. (A) Images of the tumors in the sh-LINC01535 and sh-NC groups at 4 weeks after inoculation. (B) The tumor volumes of the mice were measured every week after cell inoculation. (C) At 4 weeks after cell inoculation, the mice were euthanized and the tumors were excised and weighed. (D) Expression levels of LINC01535 in tumor tissues. (E) Expression levels of miR-761 in tumor tissues. There was no difference among the mice weights of different groups. ${ }^{* *} \mathrm{P}<0.01 \mathrm{vs}$. NC group. LINC, long intergenic non-coding RNA; sh-LINC01535, short hairpin RNA targeting LINC01535; NC, negative control; miR, microRNA.

miR-761 with clinicopathological characteristics and their prognostic value.

In conclusion, the present results indicated that LINC01535 expression is upregulated in CRC tissues and cell lines. Furthermore, LINC01535 promoted CRC proliferation, invasion and DDP resistance, partly through regulating miR-761. These results demonstrated that LINC01535 may be an important oncogenic factor in the development of CRC and may be a promising therapeutic target for CRC.

\section{Acknowledgements}

Not applicable.

\section{Funding}

No funding was received.

\section{Availability of data and materials}

The datasets used and/or analyzed during the current study are available from the corresponding author on reasonable request.

\section{Authors' contributions}

WC conceived and designed the study, and drafted the first draft of the manuscript. $\mathrm{CZ}$ and WC confirm the authenticity of all the raw data $\mathrm{WC}, \mathrm{CZ}, \mathrm{QJ}$ and $\mathrm{LC}$ conducted the experiments. $\mathrm{CZ}, \mathrm{LC}$ and QJ analyzed and collated the results. All authors reviewed, critiqued and agreed to the final submission of the manuscript. All authors read and approved the final manuscript.

\section{Ethics approval and consent to participate}

Written informed consent was obtained from every patient and the study was approved by The Ethics and Research Committees of the Affiliated Dongtai Hospital of Nantong University (Dongtai, China).

\section{Patient consent for publication}

Not applicable.

\section{Competing interests}

The authors declare that they have no competing interests. 


\section{References}

1. Zhang Y, Huang W, Yuan Y, Li J, Wu J, Yu J, He Y, Wei Z and Zhang C: Long non-coding RNA H19 promotes colorectal cancer metastasis via binding to hnRNPA2B1. J Exp Clin Cancer Res 39: 141, 2020.

2. Liang L, Chen Y, Yu Y, Pan W, Cui Y, Xu X, Peng K, Liu M, Rashid K, Hou Y and Liu T: SLC25A18 has prognostic value in colorectal cancer and represses Warburg effect and cell proliferation via Wnt signaling. Am J Cancer Res 10: 1548-1567, 2020.

3. Li Y, Liu J, Xiao Q, Tian R, Zhou Z, Gan Y, Li Y, Shu G and Yin G: EN2 as an oncogene promotes tumor progression via regulating CCL20 in colorectal cancer. Cell Death Dis 11: 604, 2020.

4. Liu Y, Sun H, Makabel B, Cui Q, Li J, Su C, Ashby CR Jr, Chen Z and Zhang J: The targeting of non-coding RNAs by curcumin: Facts and hopes for cancer therapy (review). Oncol Rep 42: 20-34, 2019.

5. Grillone K, Riillo C, Scionti F, Rocca R, Tradigo G, Guzzi PH, Alcaro S, Di Martino MT, Tagliaferri P and Tassone P: Non-coding RNAs in cancer: Platforms and strategies for investigating the genomic 'dark matter'. J Exp Clin Cancer Res 39: 117, 2020.

6. Dong L, Zhu K, Chen M, Li D, Jiang C and Chen L: Long non-coding RNA GACAT3 promotes liver cancer progression by regulating the proliferation, apoptosis and migration of tumor cells. Exp Ther Med 19: 3377-3383, 2020.

7. Zong Y, Zhang Y, Hou D, Xu J, Cui F, Qin Y and Sun X: The lncRNA XIST promotes the progression of breast cancer by sponging miR-125b-5p to modulate NLRC5. Am J Transl Res 12 3501-3511, 2020.

8. Huo W, Qi F and Wang K: Long noncoding RNA BCYRN1 promotes prostate cancer progression via elevation of HDAC11. Oncol Rep 44: 1233-1245, 2020.

9. Han Q, Li J, Xiong J and Song Z: Long noncoding RNA LINC00514 accelerates pancreatic cancer progression by acting as a ceRNA of miR-28-5p to upregulate Raplb expression. J Exp Clin Cancer Res 39: 151,2020.

10. Song Z, Zhang X, Lin Y, Wei Y, Liang S and Dong C: LINC01133 inhibits breast cancer invasion and metastasis by negatively regulating SOX4 expression through EZH2. J Cell Mol Med 23: $7554-7565,2019$

11. Lai F, Deng W, Fu C, Wu P, Cao M and Tan S: Long non-coding RNA SNHG6 increases JAK2 expression by targeting the miR-181 family to promote colorectal cancer cell proliferation. J Gene Med 22: e3262, 2020.

12. Chen B, Dragomir MP, Fabris L, Bayraktar R, Knutsen E, Liu X, Tang C, Li Y, Shimura T, Ivkovic TC, et al: The long noncoding RNA CCAT2 induces chromosomal instability through BOP1-AURKB signaling. Gastroenterology 159: 2146-2162.e33, 2020.

13. Fang Y, Zhang S, Yin J, Shen YX, Wang H, Chen XS and Tang H: LINC01535 promotes proliferation and inhibits apoptosis in esophageal squamous cell cancer by activating the JAK/STAT3 pathway. Eur Rev Med Pharmacol Sci 24 3694-3700, 2020

14. Yao X, Wu L, Gu Z and Li J: LINC01535 promotes the development of osteosarcoma through modulating miR-214-3p/KCNC4 axis. Cancer Manag Res 12: 5575-5585, 2020.

15. Song H, Liu Y, Jin X, Liu Y, Yang Y, Li L, Wang X and Li G: Long non-coding RNA LINC01535 promotes cervical cancer progression via targeting the miR-214/EZH2 feedback loop. J Cell Mol Med 23: 6098-6111, 2019.

16. Livak KJ and Schmittgen TD: Analysis of relative gene expression data using real-time quantitative PCR and the 2(-Delta Delta C(T)) method. Methods 25: 402-408, 2001
17. Filipowicz W, Bhattacharyya SN and Sonenberg N: Mechanisms of post-transcriptional regulation by microRNAs: Are the answers in sight? Nat Rev Genet 9: 102-114, 2008.

18. Chen H, Xu Z and Liu D: Small non-coding RNA and colorectal cancer. J Cell Mol Med 23: 3050-3057, 2019.

19. Xu Q, Deng F, Qin Y, Zhao Z, Wu Z, Xing Z, Ji A and Wang QJ: Long non-coding RNA regulation of epithelial-mesenchymal transition in cancer metastasis. Cell Death Dis 7: e2254, 2016.

20. Wang H, Luan H, Zhan T, Liu X, Song J and Dai H: Long non-coding RNA LINC00707 acts as a competing endogenous RNA to enhance cell proliferation in colorectal cancer. Exp Ther Med 19: 1439-1447, 2020.

21. Liu B, Pan S, Xiao Y, Liu Q, Xu J and Jia L: LINC01296/miR-26a/GALNT3 axis contributes to colorectal cancer progression by regulating O-glycosylated MUC1 via PI3K/AKT pathway. J Exp Clin Cancer Res 37: 316, 2018.

22. Zheng K and Zhang TK: LncRNA LINC00963 promotes proliferation and migration through the miR-124-3p/FZD4 pathway in colorectal cancer. Eur Rev Med Pharmacol Sci 24: 7634-7644, 2020.

23. Sun LB, Zhao SF, Zhu JJ, Han Y and Shan TD: Long noncoding RNA UCID sponges miR-152-3p to promote colorectal cancer cell migration and invasion via the $\mathrm{Wnt} / \beta$-catenin signaling pathway. Oncol Rep 44: 1194-1205, 2020.

24. Jiang Z, Li L, Hou Z, Liu W, Wang H, Zhou T, Li Y and Chen S: LncRNA HAND2-AS1 inhibits 5-fluorouracil resistance by modulating miR-20a/PDCD4 axis in colorectal cancer. Cell Signal 66: 109483, 2020.

25. Wan X, Wang C, Huang Z, Zhou D, Xiang S, Qi Q, Chen X, Arbely E, Liu CY, Du P and Yu W: Cisplatin inhibits SIRT3-deacetylation MTHFD2 to disturb cellular redox balance in colorectal cancer cell. Cell Death Dis 11: 649, 2020.

26. Zhang P, Zhao S, Lu X, Shi Z, Liu H and Zhu B: Metformin enhances the sensitivity of colorectal cancer cells to cisplatin through ROS-mediated PI3K/Akt signaling pathway. Gene 745: $144623,2020$.

27. Zhang Z, Zhang Y, Qin X, Wang Y and Fu J: FGF9 promotes cisplatin resistance in colorectal cancer via regulation of Wnt/ $\beta$-catenin signaling pathway. Exp Ther Med 19: 1711-1718, 2020.

28. Ala U: Competing endogenous RNAs, non-coding RNAs and diseases: An intertwined story. Cells 9: 1574, 2020.

29. Zhao Y, Du T, Du L, Li P, Li J, Duan W, Wang Y and Wang C: Long noncoding RNA LINC02418 regulates MELK expression by acting as a ceRNA and may serve as a diagnostic marker for colorectal cancer. Cell Death Dis 10: 568, 2019.

30. Liu Y, Chen X, Chen X, Liu J, Gu H, Fan R and Ge H: Long non-coding RNA HOTAIR knockdown enhances radiosensitivity through regulating microRNA-93/ATG12 axis in colorectal cancer. Cell Death Dis 11: 175, 2020.

31. Xiong W, Yang S, Zhang W, Chen Y and Wang F: miR-761 inhibits colorectal cancer cell proliferation and invasion through targeting HDAC1. Pharmazie 74: 111-114, 2019.

32. Zhang Q, Sui Y and Sui X: MicroRNA-761 inhibits the metastasis of gastric cancer by negatively regulating $\mathrm{Ras}$ and $\mathrm{Rab}$ interactor 1. Oncol Lett 18: 3097-3103, 2019.

33. Shi $\mathrm{C}$ and Zhang Z: miR-761 inhibits tumor progression by targeting MSI1 in ovarian carcinoma. Tumour Biol 37: 5437-5443, 2016.

This work is licensed under a Creative Commons Attribution-NonCommercial-NoDerivatives 4.0 International (CC BY-NC-ND 4.0) License. 